\title{
Applications of differential subordination for certain subclasses of meromorphically univalent functions defined by rapid operator
}

\author{
Bolineni Venkateswarlu, P. Thirupathi Reddy, Settipalli Sridevi \\ and Galla Swapna
}

Communicated by Feyzi Basar

\begin{abstract}
In this work, we investigate some applications of differential subordination for the class of meromorphic univalent functions defined by rapid operator and obtained coefficient bounds, integral representations, weighted and arithmetic mean for the class $\Sigma(A, B, \mu, \theta)$.
\end{abstract}

Keywords. Meromorphic, coefficient bound, arithmetic mean, subordination.

2020 Mathematics Subject Classification. 30C45.

\section{Introduction}

Let $\Sigma$ denote the class of functions $f$ of the form

$$
f(z)=\frac{1}{z}+\sum_{n=1}^{\infty} a_{n} z^{n}, a_{n} \geq 0
$$

which are analytic in the punctured open disk

$$
U^{*}:=\{z: z \in \mathbb{C}, 0<|z|<1\}=U \backslash\{0\} .
$$

Let $\Sigma_{s}, \Sigma^{*}(\gamma)$ and $\Sigma_{k}(\gamma)(0 \leq \gamma<1)$ denote the subclasses of $\Sigma$ that are meromorphic univalent, meromorphically starlike functions of order $\gamma$ and meromorphically convex functions of order $\gamma$ respectively. Analytically, $f \in \Sigma^{*}(\gamma)$ if and only if $f$ is of the form (1) and satisfies

$$
-\Re\left\{\frac{z f^{\prime}(z)}{f(z)}\right\}>\gamma, z \in U .
$$

Similarly, $f \in \Sigma_{k}(\gamma)$ if and only if $f$ is of the form (1) and satisfies

$$
-\Re\left\{1+\frac{z f^{\prime \prime}(z)}{f^{\prime}(z)}\right\}>\gamma, z \in U
$$


and similar other classes of meromorphically univalent functions have been extensively studied by Altintaş et al. [1], Aouf [2], Mogra et al. [5], Urlegaddi and Ganigi [9] and others (see [4, 6, 10]).

Given two functions $f$ and $g$, which are analytic in $U$, the function $f$ is said to be subordinate to $g$, written as

$$
f \prec g \text { and } f(z) \prec g(z), \quad z \in U,
$$

if there exists a Schwarz function $w$ analytic in $U$, with

$$
w(0)=0 \text { and }|w(z)|<1, \quad z \in U
$$

and such that

$$
f(z)=g(w(z)), \quad z \in U .
$$

If $g$ is univalent in $U$, then $f \prec g$ if and only if $f(0)=g(0)$ and $f(U) \subset g(U)$ ([7, p. 36]).

In [3], Athsan and Kulkarni introduced rapid operator for analytic functions and Rosy and Sunil Varma [8] modified their operator to meromorphic functions as follows.

Lemma 1.1. For $f \in \Sigma$ given by (1), $0 \leq \mu \leq 1$ and $0 \leq \theta \leq 1$, if the operator $\mathscr{S}_{\mu}^{\theta}: \Sigma \rightarrow \Sigma$ is defined by

$$
\mathscr{S}_{\mu}^{\theta} f(z)=\frac{1}{\left[(1-\mu)^{\theta} \Gamma(\theta+1)\right]} \int_{0}^{\infty} t^{\theta+1} e^{\frac{-t}{1-\mu}} f(t z) d t
$$

then

$$
\mathscr{S}_{\mu}^{\theta} f(z)=\frac{1}{z}+\sum_{n=1}^{\infty} L(n, \mu, \theta) a_{n} z^{n}
$$

where

$$
L(n, \mu, \theta)=(1-\mu)^{n+1} \frac{\Gamma(n+\theta+2)}{\Gamma(\theta+1)}
$$

and $\Gamma$ is the familiar Gamma function.

Definition 1.2. Let $A$ and $B, \quad(-1 \leq B<A \leq 1)$ be defined parameters. We say that a function $f \in \Sigma$ is in the class $\Sigma(A, B, \mu, \theta)$ if it satisfies the following subordination condition by (6)

$$
-z^{2}\left(\mathscr{S}_{\mu}^{\theta} f(z)\right)^{\prime} \prec \frac{1+A z}{1+B z}, \quad z \in U .
$$


By the definition of the differential subordination (8) is equivalent to the following condition

$$
\left|\frac{1+z^{2}\left(\mathscr{S}_{\mu}^{\theta} f(z)\right)^{\prime}}{A+B z^{2}\left(\mathscr{S}_{\mu}^{\theta} f(z)\right)^{\prime}}\right|<1, z \in U .
$$

In particular, we can write $\Sigma(1-2 \beta,-1)=,\Sigma(\beta)$, where $\Sigma(\beta)$ denotes class of the functions in $\Sigma$ satisfying the following condition:

$$
\Re\left(-z^{2}\left(\mathscr{S}_{\mu}^{\theta} f(z)\right)^{\prime}\right)>\beta, \quad 0 \leq \beta<1, z \in U
$$

The aim of this study is to determine some usual properties of the geometric function theory such as coefficient bounds, integral representation, weighted mean and arithmetic mean for the class $\Sigma(A, B, \mu, \theta)$.

\section{Coefficient bounds}

Theorem 2.1. Let the function $f$ of the form (1) be in $\Sigma$. Then the function $f$ belongs to the class $\Sigma(A, B, \mu, \theta)$ if and only if

$$
\begin{gathered}
(1-B) \sum_{n=1}^{\infty} n L(n, \mu, \theta) a_{n} \leq(A-B), \\
\text { where }-1 \leq B<A \leq 1,0 \leq \mu \leq 1 \text { and } 0 \leq \theta \leq 1 .
\end{gathered}
$$

The result is sharp for the function $f$ is given by

$$
f(z)=\frac{1}{z}+\frac{A-B}{(1-B) n L(n, \mu, \theta)} z^{n} .
$$

Proof. Assume that the condition (11) is true.

We must show that $f \in \Sigma(A, B, \mu, \theta)$ or equivalently prove that

$$
\left|\frac{1+z^{2}\left(\mathscr{S}_{\mu}^{\theta} f(z)\right)^{\prime}}{A+B z^{2}\left(\mathscr{S}_{\mu}^{\theta} f(z)\right)^{\prime}}\right|<1 .
$$


Since

$$
\begin{aligned}
\left|\frac{1+z^{2}\left(\mathscr{S}_{\mu}^{\theta} f(z)\right)^{\prime}}{A+B z^{2}\left(\mathscr{S}_{\mu}^{\theta} f(z)\right)^{\prime}}\right| & =\left|\frac{1+\left(-1+\sum_{n=1}^{\infty} n L(n, \mu, \theta) a_{n} z^{n+1}\right)}{A+B\left(-1+\sum_{n=1}^{\infty} n L(n, \mu, \theta) a_{n} z^{n+1}\right)}\right| \\
& =\left|\frac{\sum_{n=1}^{\infty} n L(n, \mu, \theta) a_{n} z^{n+1}}{A-B+B \sum_{n=1}^{\infty} n L(n, \mu, \theta) a_{n} z^{n+1}}\right| \\
& \leq\left|\frac{\sum_{n=1}^{\infty} n L(n, \mu, \theta)}{A-B+B \sum_{n=1}^{\infty} n L(n, \mu, \theta) a_{n}}\right| \\
& <1,
\end{aligned}
$$

last inequality is true by (11).

Conversely, suppose that $f \in \Sigma(A, B, \mu, \theta)$.

We must to show that the condition (11) holds. We have

$$
\begin{aligned}
& \left|\frac{1+z^{2}\left(\mathscr{S}_{\mu}^{\theta} f(z)\right)^{\prime}}{A+B z^{2}\left(\mathscr{S}_{\mu}^{\theta} f(z)\right)^{\prime}}\right|<1 \\
& \Rightarrow\left|\frac{\sum_{n=1}^{\infty} n L(n, \mu, \theta) a_{n} z^{n+1}}{A-B+B \sum_{n=1}^{\infty} n L(n, \mu, \theta) a_{n} z^{n+1}}\right|<1
\end{aligned}
$$

and since $\Re(z)<|z|$, we have

$$
\Re\left\{\frac{\sum_{n=1}^{\infty} n L(n, \mu, \theta) a_{n} z^{n+1}}{A-B+B \sum_{n=1}^{\infty} n L(n, \mu, \theta) a_{n} z^{n+1}}\right\}<1 .
$$


We choose the values of $z$ on the real axis and letting $z \rightarrow 1^{-}$, then we obtain

$$
\begin{gathered}
\left\{\frac{\sum_{n=1}^{\infty} n L(n, \mu, \theta) a_{n}}{A-B+B \sum_{n=1}^{\infty} n L(n, \mu, \theta) a_{n}}\right\}<1 \\
\Rightarrow(1-B) \sum_{n=1}^{\infty} n L(n, \mu, \theta) a_{n}<(A-B) .
\end{gathered}
$$

The result is sharp for the function $f$ is given by (12).

Corollary 2.2. Let $f \in \Sigma(A, B, \mu, \theta)$. Then

$$
a_{n} \leq \frac{(A-B)}{n(1-B) L(n, \mu, \theta)}, n \geq 1
$$

\section{Integral representation}

In the next theorem, we obtain an integral representation for $\mathscr{S}_{\mu}^{\theta} f(z)$.

Theorem 3.1. Let $f \in \Sigma(A, B, \mu, \theta)$. Then

$$
\mathscr{S}_{\mu}^{\theta} f(z)=\int_{0}^{z} \frac{(A \phi(t)-1)}{t^{2}(1-B \phi(t))} d t, \text { where }|\phi(z)|<1, z \in U .
$$

Proof. Let $f \in \Sigma(A, B, \mu, \theta)$. Letting $-z^{2}\left(\mathscr{S}_{\mu}^{\theta} f(z)\right)^{\prime}=q(z)$.

We have

$$
q(z) \prec \frac{1+A z}{1+B z}
$$

or we can write

$$
\left|\frac{q(z)-1}{B q(z)-A}\right|<1,
$$

so that consequently, we have

$$
\frac{q(z)-1}{B q(z)-A}=\phi(z),|\phi(z)|<1, z \in U .
$$

We can write

$$
-z^{2}\left(\mathscr{S}_{\mu}^{\theta} f(z)\right)^{\prime}=\frac{1-A \phi(z)}{1-B \phi(z)},
$$




$$
\begin{aligned}
-\left(\mathscr{S}_{\mu}^{\theta} f(z)\right)^{\prime} & =\frac{1}{z^{2}} \frac{1-A \phi(z)}{1-B \phi(z)} \\
\mathscr{S}_{\mu}^{\theta} f(z) & =\int_{0}^{z} \frac{(A \phi(t)-1)}{t^{2}(1-B \phi(t))} d t .
\end{aligned}
$$

Hence, the proof of theorem is completed.

\section{Linear combination}

In this section, we prove a linear combination for the class $\Sigma(A, B, \mu, \theta)$.

Theorem 4.1. Let

$$
\begin{aligned}
& f_{i}(z)=\sum_{i=1}^{k} c_{i} f_{i}(z) \in \Sigma(A, B, \mu, \theta) \\
& \text { where } \sum_{i=1}^{k} c_{i}=1 .
\end{aligned}
$$

Proof. By Theorem 2.1, we can write for every $i \in\{1,2, \cdots, k\}$,

$$
\sum_{n=1}^{\infty} \frac{n(1-B) L(n, \mu, \theta)}{A-B} a_{n, i}<1 .
$$

Therefore,

$$
\begin{aligned}
F(z) & =\sum_{i=1}^{k} c_{i}\left(z^{-1}+\sum_{n=1}^{\infty} a_{n, i} z^{n}\right) \\
& =z^{-1}+\sum_{i=1}^{k} \sum_{n=1}^{\infty} c_{i} a_{n, i} z^{n} \\
& =z^{-1}+\sum_{n=1}^{\infty}\left(\sum_{i=1}^{k} c_{i} a_{n, i}\right) z^{n}
\end{aligned}
$$

However,

$$
\begin{aligned}
& \sum_{n=1}^{\infty} \frac{n(1-B) L(n, \mu, \theta)}{A-B}\left(\sum_{i=1}^{k} c_{i} a_{n, i}\right) \\
= & \sum_{i=1}^{k}\left[\sum_{n=1}^{\infty} \frac{n(1-B) L(n, \mu, \theta)}{A-B} a_{n, i}\right] c_{i} \leq 1 .
\end{aligned}
$$


Then $F(z) \in \Sigma(A, B, \mu, \theta)$. The proof of theorem completed.

\section{Weighted mean}

Definition 5.1. Let $f$ and $g$ belongs to $\Sigma$. Then the weighted mean $h_{j}(z)$ of $f$ and $g$ is given as

$$
h_{j}(z)=\frac{1}{2}[(1-j) f(z)+(1+j) g(z)] .
$$

In the following theorem we will show the weighted mean for the class $\Sigma(A, B, \mu, \theta)$.

Theorem 5.2. If $f$ and $g$ are in the class $\Sigma(A, B, \mu, \theta)$ then the weighted mean of $f$ and $g$ are also in $\Sigma(A, B, \mu, \theta)$.

Proof. By definition of $h_{j}(z)$, we get

$$
\begin{aligned}
h_{j}(z) & =\frac{1}{2}\left[(1-j)\left(z^{-1}+\sum_{n=1}^{\infty} a_{n} z^{n}\right)+(1+j)\left(z^{-1}+\sum_{n=1}^{\infty} b_{n} z^{n}\right)\right] \\
& =z^{-1}+\frac{1}{2} \sum_{n=1}^{\infty}\left[(1-j) a_{n}+(1+j) b_{n}\right] z^{n} .
\end{aligned}
$$

Since $f, g \in \Sigma(A, B, \mu, \theta)$, by Theorem 2.1, we must prove that

$$
\begin{aligned}
& \sum_{n=1}^{\infty} n(1-B) L(n, \mu, \theta)\left[\frac{1}{2}(1-j) a_{n}+\frac{1}{2}(1+j) b_{n}\right] \\
& =\frac{1}{2}(1-j)(1-B) \sum_{n=1}^{\infty} n L(n, \mu, \theta) a_{n}+\frac{1}{2}(1+j)(1-B) \sum_{n=1}^{\infty} n L(n, \mu, \theta) b_{n} \\
& \leq \frac{1}{2}(1-j)(A-B)+\frac{1}{2}(1+j)(A-B) \\
& \leq(A-B) .
\end{aligned}
$$

Hence the proof of theorem is completed.

\section{Arithmetic mean}

Definition 6.1. Let $f_{1}(z), f_{2}(z), \cdots, f_{k}(z) \in \Sigma(A, B, \mu, \theta)$. Then the arithmetic mean $h(z)$ of $f_{i}(z)$ is given by $h(z)=\frac{1}{k} \sum_{i=1}^{k} f_{i}(z)$. 
Next, we will prove the arithmetic mean for the class $\Sigma(A, B, \mu, \theta)$.

Theorem 6.2. If $f_{1}(z), f_{2}(z), \cdots, f_{k}(z)$ are in the class $\Sigma(A, B, \mu, \theta)$ then the arithmetic mean $h(z)$ of $f_{i}(z)$ is given by

$$
h(z)=\frac{1}{k} \sum_{i=1}^{k} f_{i}(z)
$$

is also in the class $\Sigma(A, B, \mu, \theta)$.

Proof. We have for $h(z)$ by definition

$$
\begin{aligned}
h(z) & =\frac{1}{k} \sum_{i=1}^{k}\left[z^{-1}+\sum_{n=1}^{\infty} a_{n, i} z^{n}\right] \\
& =z^{-1}+\sum_{n=1}^{\infty}\left[\frac{1}{k} \sum_{i=1}^{k} a_{n, i}\right] z^{n} .
\end{aligned}
$$

Since $f_{i}(z) \in \Sigma(A, B, \mu, \theta)$, for every $i \in\{1,2, \cdots, k\}$, by using Theorem 2.1, we prove that

$$
\begin{aligned}
(1-B) \sum_{n=1}^{\infty} n L(n, \mu, \theta)\left(\frac{1}{k} \sum_{i=1}^{k} a_{n, i}\right) & =\frac{1}{k} \sum_{i=1}^{k}\left(\sum_{n=1}^{\infty} n(1-B) L(n, \mu, \theta) a_{n, i}\right) \\
& \leq \frac{1}{k} \sum_{i=1}^{k}(A-B) .
\end{aligned}
$$

The proof of theorem completed.

Acknowledgments. The authors express their sincere thanks to the esteemed referee(s) for their careful readings, valuable suggestions and comments, which helped them to improve the presentation of the paper.

\section{Bibliography}

[1] O. Altintaş, H. Irmak and H. M. Srivastava, A family of meromorphically univalent functions with positive coefficients, PanAmer. Math. J. 5(1) (1995), 75-81.

[2] M. K. Aouf, On a certain class of meromorphic univalent functions with positive coefficients, Rend. Mat. Appl. 11 (1991), 209-219. 
[3] W. G. Atshan and R. H. Buti, Fractional calculus of a class of univalent functions with negative coefficients defined by Hadamard product with rapid-operator, Eur. J. Pure Appl. Math. 4(2) (2011), 162-173.

[4] M. Darus, Meromorphic functions with positive coefficients, Int. J. Math. Math. Sci. 6 (2004), 319-324.

[5] M. L. Mogra, T. R. Reddy and O. P. Juneja, Meromorphic univalent functions with positive coefficients, Bull. Aust. Math. Soc. 32(2) (1985), 161-176.

[6] S. Owa and N. N. Pascu, Coefficient inequalities for certain classes of meromorphically starlike and meromorphically convex functions, J. Inequal. Pure Appl. Math. 4(1) (2003), Article 17, 1-6.

[7] Ch. Pommerenke, Univalent Functions, Vanderhoeck and Ruprecht, Göttingen, 1975.

[8] T. Rosy S. Sunil Varma, On a subclass of meromorphic functions defined by Hilbert space operator, Geometry (2013), Article 671826, 1-4.

[9] B. A. Uralegaddi and M. D. Ganigi, A certain class of meromorphically starlike functions with positive coefficients, Pure Appl. Math. Sci. 26(1-2) (1987), 75-81.

[10] B. Venkateswarlu, P. Thirupathi Reddy and N. Rani, Certain subclass of meromorphically uniformly convex functions with positive coefficients, Mathematica 61(84)(1) (2019), 85-97.

Received July 10, 2020; revised January 20, 2021; accepted November 23, 2021.

\section{Author information}

Bolineni Venkateswarlu, Department of Mathematics, GSS, GITAM University,

Bengaluru Rural, India.

E-mail: bvlmaths@gmail.com

P. Thirupathi Reddy, Department of Mathematics, Kakatiya University, Warangal-506 009, Telangana, India.

E-mail: reddypt2@gmail.com

Settipalli Sridevi, Department of Mathematics, GSS, GITAM University, Bengaluru

Rural, India.

E-mail: siri_settipalli@yahoo.co.in

Galla Swapna, Department of Mathematics, GSS, GITAM University, Bengaluru Rural, India.

E-mail: swapna.priya38@gmail.com 